'Programa de trasplante hematopoyético del adulto. Facultad de Medicina, Pontificia Universidad Católica de Chile, Red de salud UC Christus. Santiago, Chile.

Trabajo no recibió financiamiento. Los autores declaran no tener conflictos de interés.

Recibido el 23 de octubre de 2020, aceptado el 23 de noviembre de 2020 .

Correspondencia a: Dr. Mauricio Sarmiento M. Lira 85, 4to piso. Santiago, Chile. msarmiento@med.puc.cl

\section{Resultados a largo plazo de una cohorte chilena: la edad del paciente no incide en el resultado del trasplante alogénico de precursores hematopoyéticos para leucemia mieloide aguda}

\author{
MAURICIO SARMIENTO ${ }^{1}$, PATRICIO ROJAS ${ }^{1}$, \\ NICOLÁS TRIANTAFILO ${ }^{1}$, JAMES CAMPBELL ${ }^{1}$, \\ MARÍA JOSÉ GARCÍA ${ }^{1}$, MAURICIO OCQUETEAU ${ }^{1}$, \\ VICENTE SANDOVAL ${ }^{1}$, ANDRÉS ROJAS ${ }^{1}$, JOSÉ TOMÁS GAZMURI ${ }^{1}$, \\ GONZALO GUERRERO ${ }^{1}$, MAXIMILIANO VERGARA ${ }^{1}$, PABLO BERTIN $^{1}$, \\ PABLO RAMÍREZ ${ }^{1}$, VERÓNICA JARA ${ }^{1}$, CATHERINE GUTIÉRREZ ${ }^{1}$, \\ KATHERINE SOTO ${ }^{1}$, SILVANA ARELLANO ${ }^{1}$, ISABEL PIZARRO ${ }^{1}$, \\ CARLA LORCA ${ }^{1}$, ELIZABETH RIVERA ${ }^{1}$, ELVIRA ÁLVAREZ $^{1}$, \\ MARCIA VALDÉS ${ }^{1}$, JAIME PEREIRA ${ }^{1}$, FRANCISCO BARRIGA ${ }^{1}$
}

\section{Age does not affect the outcome of allogeneic hematopoietic precursor transplantation for acute myeloid leukemia}

Background: In our country, transplantation centers differ in the age limit for allogeneic hematopoietic transplantation (ALOHT). In our program, transplants with age-adjusted conditioning are performed in patients until 70 years old. Currently more than $60 \%$ of ALOHT reported to the Center for International Bone Marrow Transplantation Research (CIBMTR) are performed in patients older than 40 years. Aim: To report our experience with ALOHT in acute myelogenous leukemia (AML), analyzing patient age at transplantation in different periods and transplant results in different age groups. Material and Methods: $A$ retrospective analysis of the database of adult hematopoietic transplants in $A M L$ patients was performed. Demographic data, disease characteristics, transplant data, survival and relapse times, and mortality were collected. Results: In our program, 1030 transplants were performed in adults and 119 ALOHT were performed in AML patients, between 1990 and 2020. The median age of patients in all periods was 41 years, (range 16-69). The median age was 33 and 45 years, in the periods 1990-2000 and 2000-2020 respectively $(p<0.01)$. Seventy-eight patients received myeloablative conditioning (median age 44 years) and 41 reduced intensity conditioning (median age 53 years). Five-year overall survival was $44.6 \%$ (confidence intervals (CI) 41-48). Non relapse mortality of all periods was 19\% (CI $17-40 \%$ ) and relapse rate was $17 \%$ (CI 16-22). No difference in five years overall survival among patients younger than 40, 41 to 50 and over 51 years was observed. Conclusions: Overall Survival, non-relapse mortality and relapse rate were similar in younger and older patients in our program and similar to those previously reported in other centers.

(Rev Med Chile 2021; 149: 22-29)

Key words: Leukemia; Survival; Transplantation, Homologous. 
L a leucemia mieloide aguda (LMA) es uno de los cánceres hematológicos más frecuentes y cerca de 50\% tiene más de 60 años al diagnóstico ${ }^{1}$. El trasplante alogénico de precursores hematopoyéticos es, a la fecha, el único tratamiento potencialmente curativo para los pacientes de riesgo intermedio o alto y suelen aplicarse estrictos criterios etarios de selección que puede apartar a muchos individuos de su beneficio ${ }^{2}$. En las últimas décadas la optimización del proceso de trasplante y de la aplicabilidad de éste acorde no solo a la edad cronológica sino en torno al funcionamiento fisiológico del individuo con leucemia han permitido aumentar progresivamente la edad de corte para aplicar al trasplante, siendo incluso en USA o Europa hasta los 75 años ${ }^{3,4}$. Según datos reportados por el Ministerio de Salud, en Chile la mediana de edad al debut de la leucemia es de 48 años y se requieren tratar aproximadamente 400 a 500 personas cada año por leucemia aguda. $\mathrm{Si}$ esto se proyecta a la probabilidad de que el $30 \%$ de los individuos con LMA precisarán trasplante, por lo menos 150 pacientes al año deberían ser trasplantados. Lamentablemente este cálculo del modelo ideal está lejos de la realidad, puesto que no todos los pacientes que requieren la realización de un trasplante de consolidación acceden finalmente a la terapia. Los motivos son diversos, e incluyen la no disponibilidad de donante emparentado idóneo, la refractariedad de la enfermedad que impide llegar en condiciones óptimas al trasplante, la fragilidad individual o la imposibilidad de aplicar el trasplante por protocolos definidos localmente ${ }^{5}$. En este sentido la guía ministerial de 2016 ofrece trasplante alogénico a menores de 40 años, al mismo tiempo que en programas universitarios o del ámbito privado del país este no es un factor limitante, pudiendo elevarse incluso hasta los 70 años. El uso de tratamientos preparatorios de intensidad reducida, con significativamente menor toxicidad que los tratamientos mieloablativos han permitido trasplantar pacientes cada vez de mayor edad con resultados comparables a los pacientes más jóvenes ${ }^{6}$. Por otra parte, los donantes familiares haploidénticos $(50 \%)$ han permitido extender el trasplante a pacientes sin hermanos $100 \%$ compatibles $^{7}$. En último término la disponibilidad de donantes no emparentados de registros nacionales e internacionales ha permitido encontrar donantes en aquellos sin familiares disponibles. El resultado es que casi todo paciente tiene acceso a trasplante independiente de su edad. En este trabajo presentamos nuestra experiencia en trasplante alogénico en pacientes afectos por LMA con énfasis en los resultados obtenidos en diferentes grupos etareos.

\section{Metodología}

La información para el análisis de esta investigación fue obtenida de la base de datos del programa de trasplante hematopoyético del adulto y de la historia clínica electrónica y en archivo físico, obteniendo datos de todos los pacientes sometidos a AloTH desde el inicio del programa en 1993 a la fecha.

La información obtenida fue: datos demográficos, estadio de enfermedad el trasplante, tiempos de sobrevida libre de progresión y global, incidencia de enfermedad de injerto vs huésped, mortalidad por recidiva u otras causas relacionadas al trasplante. Analizamos la distribución etaria y los resultados por período entre las décadas 1990 a 1999,2000 a 2009 y 2010 a 2020.

\section{Trasplante hematopoyético: donante y paciente}

El procedimiento en extenso de valoración pretrasplante fue reportado previamente ${ }^{6,7}$. Al diagnóstico o en las fases iniciales del tratamiento del paciente evaluamos la compatibilidad de los potenciales donantes emparentados por medio de genotipificación de HLA A, B, C, DRB buscando un hermano $100 \%$ compatible. De no hallarlo, se estudiaron los familiares $50 \%$ compatibles, en los que se buscan anticuerpos anti HLA. Una vez fue seleccionado el donante idóneo evaluamos la serología del mismo ante enfermedades infecciosas tales virus hepatotropos, de la familia herpes viridae, toxoplasmosis, sarampión, rubéola, VIH, HTLV 1 entre otros. Los precursores hematopoyéticos del donante fueron movilizados mediante el uso de filgrastim por 5 días al cabo de los cuales procedimos a aféresis por vía periférica para obtener un mínimo de 2x10e6 células CD34 por kilo de peso del paciente.

Los pacientes recibieron tratamiento de quimioterapia al diagnóstico o recidiva con el objeto de obtener una remisión completa, después de lo cual procedieron al trasplante. El tratamiento preparatorio (condicionamiento) fue realizado de acuerdo a la enfermedad de base y la edad. Previo 
al año 2000 todos los pacientes recibieron esquemas mieloablativos con busulfan o irradiación corporal total y ciclofosfamida. A partir de ese año los pacientes mayores de 40 años recibieron esquemas de intensidad reducida. En los últimos años se incorporó un esquema orientado a pacientes con LMA refractaria usando clofarabina como puente al trasplante ${ }^{8}$.

\section{Profilaxis de enfermedad de injerto vs huésped (EICH)}

La profilaxis usada se determinó de acuerdo al tipo de donante. Los pacientes con donante emparentado HLA idéntico usamos ciclosporina y metotrexate. En pacientes con donante no emparentado HLA idéntico usamos tacrolimus y ATG y en pacientes con donante haploidéntico ciclofosfamida $50 \mathrm{mg} / \mathrm{g}$ por en días 3 y 4 seguidas de tacrolimus y micofenolato

\section{Medidas de soporte}

Durante el período de aplasia, los pacientes recibieron transfusiones para mantener plaquetas $>10.000-20.000 /$ uL y hemoglobina $>7-8 \mathrm{~g} \%$ y recibieron filgrastim hasta lograr recuentos de neutrófilos $>500 / \mathrm{uL}$. El tratamiento antimicrobiano preventivo consistió en aciclovir y fluconazol que fue reemplazado por posaconazol en 2016. Ante neutropenia febril, se realizó estudio y tratamiento según guías institucionales, con hemocultivo, urocultivo y radiografía de tórax seguido de tratamiento antibiótico con cefalosporinas de $3^{\text {ra }}$ generación, aminoglucósido y vancomicina. En caso de persistencia de fiebre evaluamos al paciente con tomografía computada (TAC) de cavidades paranasales, tórax, abdomen y pelvis y tests para infección fúngica invasora El tratamiento antimicrobiano fue modificado según los hallazgos radiológicos o microbiológicos. En caso de reactivación de citomegalovirus, se inició terapia preventiva con ganciclovir o foscarnet. Todos los pacientes recibieron tratamiento analgésico opiáceo en caso de mucositis grado 2 a 4 graves y nutrición parenteral según necesidad.

\section{Definiciones de complicaciones post trasplante}

La EICH se definió según el tiempo de aparición y desarrollo de las características clínicas, aguda cuando apareció antes de los 100 días y crónica después de los mismos. Los pacientes fueron censados en el momento de la recaída, falle- cimiento o último control para determinar sobrevida global y libre de eventos, y en el momento de fallecimiento por otra causa que la leucemia para estimar la mortalidad relacionada a trasplante. $\mathrm{La}$ recaída se definió como la evidencia de aparición de blastos leucémicos en sangre o médula ósea post trasplante.

\section{Análisis estadístico}

La prueba de Shapiro-Wilk evaluó la normalidad de las variables cuantitativas. Las características demográficas y basales fueron presentadas utilizando media, porcentaje y rangos. Las comparaciones entre las variables se realizaron con el método de Chi cuadrado. Las probabilidades de SG se calcularon con el método de Kaplan-Meier y las comparaciones con la prueba de log-rank. Los análisis de recaída, mortalidad no relacionada a recaída y EICH se realizaron en un marco de riesgos competitivos mediante el estimador no paramétrico de incidencia acumulada. Para estimar el efecto de la edad en la SG, se ajustó el modelo de Kaplan-Meier de acuerdo a los rangos de edad comprendidos en menores de 40 años, entre 41 y 50 años y mayores de 51 años. El efecto de los acontecimientos que tuvieron lugar durante el seguimiento y después del trasplante, como la EICH aguda, fueron analizados como una covariable dependiente del tiempo. El software usado fue SPSS.V.15 (IBM Software, USA) y Prisma Software V 6.0.1 (GraphPad software, USA). Las diferencias se consideraron significativas para valores de $\mathrm{p}<0,05$ con intervalos de confianza (IC) del 95\%.

\section{Aspectos éticos}

Todos los pacientes firmaron consentimiento informado aprobado por el comité de ética de nuestra institución y en donde se detalla explícitamente la autorización al uso de datos para investigación retrospectiva protegiendo la confidencialidad.

\section{Resultados}

\section{Características demográficas de los pacientes}

Durante el período de análisis se realizaron 119 trasplantes en pacientes con leucemia mieloide aguda. La mediana de edad fue de 41 años con rango entre 16 a 69 años y $43 \%$ fueron mujeres. 
La mayoría de los pacientes ingresaron en segunda o tercera remisión completa o enfermedad refractaria. Usamos esquema mieloablativo en un $70 \%$ de los pacientes. Otras características se muestran en la Tabla 1. Se analizaron los cambios en la

Tabla 1. Características de los pacientes previo al trasplante

\begin{tabular}{|c|c|c|}
\hline $\begin{array}{l}\text { Población } \\
\text { analizada }\end{array}$ & $n=119$ & \\
\hline Edad promedio & $\begin{array}{l}41 \text { años rango 16-69 } \\
\text { Percentil 25: } \\
\text { Percentil 50: } \\
\text { Percentil 75: } \\
\text { Edad máxima: }\end{array}$ & $\begin{array}{l}16 \text { años } \\
39 \text { años } \\
55 \text { años } \\
69 \text { años }\end{array}$ \\
\hline Género & $\begin{array}{l}\text { Femenino } \\
\text { Masculino }\end{array}$ & $\begin{array}{l}39(43 \%) \\
80(57 \%)\end{array}$ \\
\hline $\begin{array}{l}\text { Alteraciones } \\
\text { citogenéticas }\end{array}$ & $\begin{array}{l}\text { Sin información: } \\
\text { Cariotipo normal: } \\
\text { Complejo: } \\
\text { Deleción cromosoma 7: } \\
\text { Deleción }(11 \text { q;23): } \\
\text { Inversión cromosoma } 16 \\
\text { Trisomia 8: }\end{array}$ & $\begin{aligned} \mathrm{n}= & 48 \\
\mathrm{n}= & 48 \\
\mathrm{n}= & 8 \\
\mathrm{n}= & 5 \\
\mathrm{n}= & 2 \\
\mathrm{n}= & 4 \\
\mathrm{n}= & 4\end{aligned}$ \\
\hline $\begin{array}{l}\text { Alteraciones } \\
\text { moleculares } \\
\text { por PCR }\end{array}$ & $\begin{array}{l}\text { Desconocido o no técnica } \\
\text { diagnostica no disponible } \\
\text { al momento del trasplante } \\
\text { t(8:21): } \\
\text { Inversión 16: } \\
\text { FLT3 mutado: } \\
\text { NPM1: } \\
\text { t(15:17) }\end{array}$ & $\begin{array}{l}\mathrm{n}=108 \\
\mathrm{n}=3 \\
\mathrm{n}=3 \\
\mathrm{n}=3 \\
\mathrm{n}=2 \\
\mathrm{n}=1\end{array}$ \\
\hline $\begin{array}{l}\text { Estatus al } \\
\text { trasplante }\end{array}$ & $\begin{array}{l}\text { Primer remisión completa } \\
\text { Segunda remisión completa } \\
\text { Tercer remisión completa } \\
\text { Refractaria }\end{array}$ & $\begin{aligned} \mathrm{n} & =58 \\
\mathrm{n} & =34 \\
\mathrm{n}= & 8 \\
\mathrm{n}= & 19\end{aligned}$ \\
\hline
\end{tabular}

edad de los trasplantados a través de la historia del programa encontrando que en los últimos 10 años la edad subió en promedio 13 años como se muestra en la Tabla $2(\mathrm{p}=0,0034)$.

\section{Características del injerto y resultados derivados}

La celularidad infundida en promedio fue de 5,9 x $10 \exp 6$ CD34/kg. La mayoría de los pacientes tuvieron injerto de granulocitos al día 20 e injerto plaquetario al día 26. La incidencia acumulada de enfermedad de injerto vs huésped aguda y crónica global se muestra en las Tablas 3 y 4 donde se resumen los resultados obtenidos del proceso del trasplante.

\section{Sobrevida global}

La sobrevida global de los pacientes del estudio a 5 años fue de $44,6 \%$. En el análisis realizado por grupos etareos se encontró que a 5 años los pacientes menores de 40 , de 41 a 50 y mayores de 51 años tuvieron una sobrevida global de 45,41 y $39 \%$ respectivamente (Figura 1 ).

\section{Mortalidad}

Cincuenta y cinco pacientes fallecieron durante el período estudiado con una mortalidad de $44 \%$. El $19 \%$ fue por recaída $(\mathrm{n}=23)$ y $26 \%$ por otras causas, incluyendo sepsis $(n=19)$, GVHD refractario $(n=10)$, y accidentes vasculares hemorrágicos $(\mathrm{n}=3)$. Al analizar la mortalidad no relacionada a recaída de acuerdo a la edad no se encontraron diferencias significativas como se muestra en las Tabla 5 y en la Figura 2. Tampoco se encontraron diferencias significativas en cuanto a la recaída y la incidencia de enfermedad de injerto vs huésped como se muestra en las Figuras 3 y 4.

Tabla 2. Análisis comparativo de las edades de los trasplantados en diferentes épocas de actividad del programa de trasplante

\begin{tabular}{|c|c|c|c|c|}
\hline Edad & $\begin{array}{l}\text { Actividad entre } \\
\begin{array}{c}1990 \text { y } 1999 \\
n=10\end{array}\end{array}$ & $\begin{array}{l}\text { Actividad entre } \\
2000 \text { y } 2009 \\
n=34\end{array}$ & $\begin{array}{l}\text { Actividad entre } \\
\begin{array}{c}2010 \text { y } 2020 \\
n=75\end{array}\end{array}$ & $\begin{array}{c}\text { Significancia estadística } \\
\text { (test no paramétrico } \\
\text { de ANOVA) }\end{array}$ \\
\hline $\begin{array}{l}\text { Mínima } \\
\text { Percentil 25\% }\end{array}$ & $\begin{array}{c}17 \mathrm{a} \\
22.75 \mathrm{a}\end{array}$ & $\begin{array}{l}18 \mathrm{a} \\
30 \mathrm{a}\end{array}$ & $\begin{array}{c}16 a \\
34.5 a\end{array}$ & \multirow{3}{*}{$p=0,00035$} \\
\hline $\begin{array}{l}\text { Mediana } \\
\text { Percentil 75\% }\end{array}$ & $\begin{array}{l}33.5 \mathrm{a} \\
37.5 \mathrm{a}\end{array}$ & $\begin{array}{l}44 a \\
49 a\end{array}$ & $\begin{array}{c}45.5 a \\
58 a\end{array}$ & \\
\hline Máxima & $57 a$ & $55 a$ & $69 a$ & \\
\hline
\end{tabular}


Tabla 3. Características del trasplante

\begin{tabular}{|ll|}
\hline Acondicionamiento usado & 1900-1999: $n=10$ \\
& CyTBI: 10 \\
& 200-2009: $n=34$ \\
& Mieloablativos (BUCY $n=28$, Etoposido-CYTB $n=2, B \cup C Y$ TBI $n=4)$ \\
& $2010-2020 n=75$ \\
& Mieloablativos (CyTBI $n=38$, BUCY $n=1$, clofarabine $n=4)$ ) \\
& Intensidad reducida (FLUMEL $n=25$, FLUBU $n=7$ ) \\
& HAPLO 41 \\
Tipo de donante & DNR 7 \\
& Donante emparentado HLA idéntico: 71 \\
& Ciclosporina methotrexate: 78 \\
Profilaxis de EICH & Tacrolimus/micofenolato: 41 \\
& PTCY: 41 \\
Fuente progenitores & Sangre periférica por aféresis: 116 \\
& Médula ósea: 3 \\
Aféresis realizadas & Una aféresis: 90 \\
& Dos aféresis: 20 \\
& 3 o más aféresis: 6 \\
& En 3 casos se usó plerixafor en el donante \\
\hline
\end{tabular}

Esquemas mieloablativos. CYTBI: $60 \mathrm{mg} / \mathrm{kg} /$ día por dos días con o sin busulfán oral asociado y TBI 1.320 cGY dividido en 3 a 4 días. BUCY: busulfán VO $1 \mathrm{mg} / \mathrm{kg} / 6 \mathrm{~h}$ por 4 días y ciclofosfamida $60 \mathrm{mg} / \mathrm{kg}$ día por 2 días. Etopósido-CYTBI: Etopósido 200 $\mathrm{mg} / \mathrm{m}^{2} /$ día por 4 días junto con CYTBI. Esquemas de intensidad reducida: FLU-MEL: Fludarabina IV $40 \mathrm{mg} / \mathrm{m}^{2} \mathrm{cada}$ día por 5 días con melfalán 100 o 140 mg/m² dosis única. FLU-Bu: Fludarabina IV 40 mg/m² cada día por 5 días con busulfan 1 mg/ $\mathrm{kg} /$ día por 2 días. Bridge: clofarabina $30 \mathrm{mg} / \mathrm{m}^{2} /$ día por 5 días. PTCY: ciclofosfamida post trasplante $50 \mathrm{mg} / \mathrm{kg} / \mathrm{d}$ por 2 días.

Tabla 4. Resultados del trasplante

\begin{tabular}{|c|c|}
\hline $\begin{array}{l}\text { Celularidad infundida } \\
\text { (CD34 x } 10 \text { exp 6/Kg peso) }\end{array}$ & $\begin{array}{l}5,9 \times 10 \exp 6 \mathrm{CD} 34 / \mathrm{kg} \\
\text { rango } 2-14\end{array}$ \\
\hline $\begin{array}{l}\text { Día de prendimiento } \\
\text { granulocítico }\end{array}$ & 20 rango $16-45$ \\
\hline $\begin{array}{l}\text { Día de prendimiento } \\
\text { plaquetario }\end{array}$ & 26 rango $16-120$ \\
\hline $\begin{array}{l}\text { Incidencia acumulada de } \\
\text { EICH aguda a } 100 \text { días } \\
1-2 \\
3-4\end{array}$ & $\begin{array}{l}42 \% \\
22 \%\end{array}$ \\
\hline $\begin{array}{l}\text { Incidencia acumulada de } \\
\text { EICH crónica a } 2 \text { años } \\
\text { Limitada } \\
\text { Extensa }\end{array}$ & $\begin{array}{l}40 \% \\
18 \%\end{array}$ \\
\hline
\end{tabular}

\section{Estado de la enfermedad y resultado del trasplante}

Con respecto al estado de la enfermedad al momento del trasplante encontramos que aquellos pacientes que fueron trasplantados en 1er remisión completa tuvieron a 5 años una sobrevida

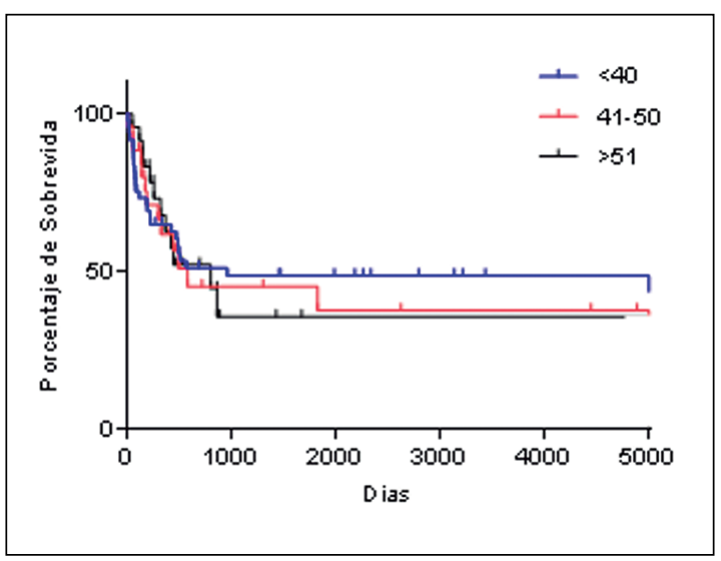

Figura 1. Sobrevida global de todos los trasplantados por leucemia mieloide aguda de acuerdo a la edad.

global de 65\%, mientras que en los pacientes que fueron trasplantados en $2 \mathrm{da}$ o posterior remisión completa fue $42 \%$ y en los pacientes que accedieron al trasplante con enfermedad activa la sobrevida fue de $20 \%$, con sobrevida prolongada en 4 de 19 pacientes (Figura 5). 
Tabla 5. Análisis de mortalidad según el período de funcionamiento del programa

\begin{tabular}{|c|c|c|c|}
\hline $\begin{array}{l}\text { Período del } \\
\text { programa }\end{array}$ & $\begin{array}{l}\text { Mortalidad } \\
\text { relacionada a } \\
\text { trasplante }\end{array}$ & $\begin{array}{l}\text { Mortalidad por recaída } \\
\text { o progresión de la } \\
\text { enfermedad }\end{array}$ & $\begin{array}{l}\text { Mortalidad por otras } \\
\text { causas no relacionadas a } \\
\text { recaída o trasplante }\end{array}$ \\
\hline $\begin{array}{l}1990-1999 \\
n=5 \text { ( } 50 \% \text { del total } \\
\text { del período) }\end{array}$ & $\begin{array}{c}40 \% \\
\text { Sepsis } n=2 \\
\text { ElCH } n=2\end{array}$ & $\begin{array}{c}10 \% \\
\text { Recaída } n=1\end{array}$ & No \\
\hline $\begin{array}{l}2000-2009 \\
\mathrm{n}=18(55 \% \text { del total } \\
\text { del período) }\end{array}$ & $\begin{array}{c}32 \% \\
\text { Sepsis } n=8 \\
\text { EICH } n=3\end{array}$ & $\begin{array}{c}19 \% \\
\text { Recaída } n=6\end{array}$ & $\begin{array}{c}4 \% \\
\text { Accidente vascular } \\
\text { encefálico } n=1\end{array}$ \\
\hline $\begin{array}{l}2010-2020 \\
\mathrm{n}=32(42 \% \text { del total } \\
\text { del período) }\end{array}$ & $\begin{array}{c}17 \% \\
\text { Sepsis } n=9 \\
\text { EICH } n=4\end{array}$ & $\begin{array}{c}22 \% \\
\text { Recaída } n=17\end{array}$ & $\begin{array}{c}2 \% \\
\text { Accidente vascular } \\
\text { encefálico } n=2\end{array}$ \\
\hline Significancia estadística & $p=0,0005$ & $p=0, .006$ & $p=0,9$ \\
\hline
\end{tabular}

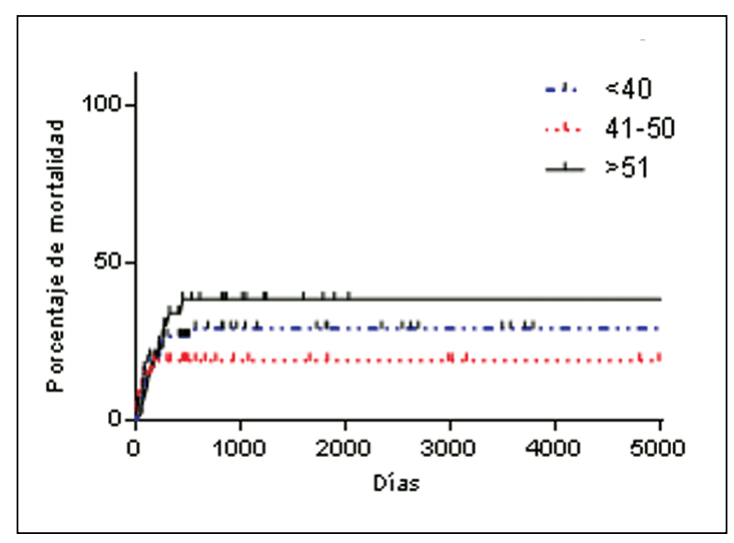

Figura 2. Mortalidad no relacionada a recaída de los pacientes con LMA de acuerdo a la edad.

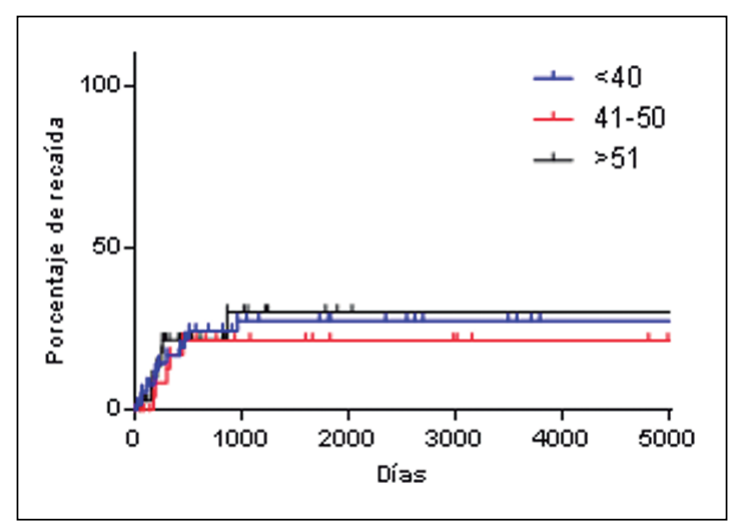

Figura 4. Recaída en pacientes con leucemia mieloide aguda tratados con trasplante hematopoyético alogénico de acuerdo con la edad al momento del trasplante.

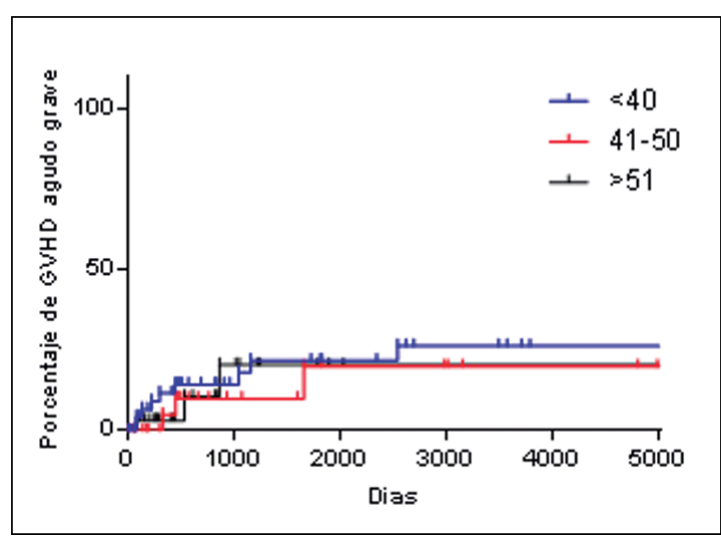

Figura 3. Incidencia de enfermedad de injerto vs huésped grave en pacientes con leucemia mieloide aguda de acuerdo a la edad.

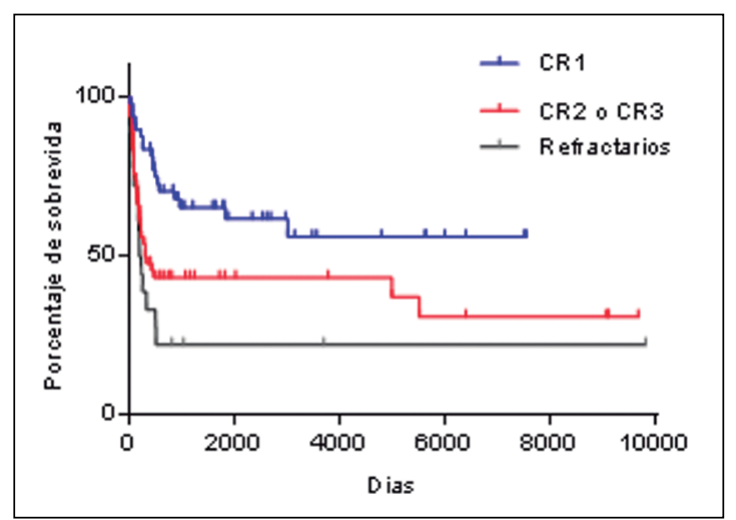

Figura 5. Sobrevida global en los pacientes según el estado de la enfermedad al momento del trasplante. 


\section{Discusión}

Nuestro análisis permite observar que con los ajustes apropiados en la terapia de acondicionamiento se obtuvieron resultados similares a los reportados en grandes series de pacientes en centros de referencia con tasas de morbilidad y mortalidad aceptables ${ }^{9}$. Es destacable además que en los últimos años de actividad del programa, la sobrevida y tasa de remisión y complicaciones del procedimiento han mejorado sostenidamente. Esto puede ser debido al rol de varios factores como: a) mejores medidas de soporte antibiótico, antifúngico y de cuidados intensivos, b) mejor precisión en la selección de donantes, C) incorporación de los haplo donantes como donantes alternativos al familiar HLA idéntico y d) adecuación de la intensidad del acondicionamiento en función de la edad. Así, pudimos determinar que la aplicación de protocolos de intensidad reducida a pacientes mayores de 40 años, permite realizar ALOTH con resultados comparables a los obtenidos en los individuos jóvenes lo cual nos permite afirmar que la edad cronológica no es un factor determinante para la realización del trasplante. Es importante reconocer que la limitación de nuestro análisis está dada por la naturaleza retrospectiva, pero que dado el tiempo de funcionamiento del programa se puede apreciar el comportamiento de nuestra población en datos del mundo real en donde nuestra principal problemática es la aplicación del trasplante y la extensión de su uso en la rango de edad que así lo requieran.

Hace varios años a nivel global es aceptado que el trasplante es un tratamiento "standard de cuidado" en individuos con leucemia mieloide aguda ${ }^{10}$. Ante la creciente evidencia disponible se han realizado meta análisis que han confirmado lo reportado en numerosos estudios individuales mostrando que los pacientes sometidos a trasplante hematopoyético por leucemia tienen mejor sobrevida comparativamente con aquellos no trasplantados, teniendo presente que la limitante de la mayoría de los estudios fueron realizados con la randomización natural al no tener donante disponible $^{11}$. A nivel global se ha ido incrementando la edad límite para realizar los trasplantes ya que esta enfermedad es habitual en adultos mayores. Así, se ha descrito que la aplicación racional en el ajuste en la intensidad del tratamiento, permite realizar el trasplante en pacientes cada vez más mayores con resultados similares, fundamentado esto en la asignación selectiva del tipo de acondicionamiento para el trasplante según el performance status y una minuciosa evaluación pre trasplante por sistemas cardiopulmonar, hepático, renal, digestivo, etc ${ }^{12}$. Recientemente, la sociedad Europea de trasplante de médula ósea realizó un análisis de 713 pacientes mayores de 70 años comparados con 16.000 pacientes entre 50 y 69 años trasplantados por LMA entre 2001 y 2014. Una alta proporción de los pacientes mayores fueron hombres, con leucemia secundaria, llegaron a trasplante con enfermedad activa y recibieron trasplantes no relacionados. A pesar de todos estos factores adversos, se pudo constatar que la incidencia de EICH fue similar en los dos grupos y que la sobrevida global fue de 34 vs 50\%, la cual es bastante similar a lo que estamos reportando en nuestro trabajo ${ }^{13}$. Otro estudio reciente comparó los resultados del trasplante en pacientes entre 60 y 77 años tratados con alotrasplante en norteamérica, reportó que la media de edad fue de 67a y que si bien la mortalidad relacionada a trasplante fue alta en principio, luego del primer año de seguimiento bajó considerablemente a valores similares a los de pacientes jóvenes ${ }^{14}$.

Si bien, la edad máxima de trasplante que se usa en centros europeos y norteamericanos ronda los 75 años esto es de difícil aplicabilidad en nuestro medio $^{15}$; sin embargo, nuestros datos apoyan un posible ajuste en el límite hasta los 65-70 años basado en valoración multidisciplinaria que permita establecer que el individuo está en condiciones para soportar el tratamiento independiente de la edad y con aplicación de esquemas de acondicionamiento ajustados. Así podemos concluir que durante las 3 décadas de funcionamiento de nuestro programa de trasplante se ha aumentado progresivamente la edad de los individuos trasplantados con morbimortalidad del procedimiento sin diferencias, pudiendo reproducir los resultados reportados en hemisferio norte.

\section{Referencias}

1. Cornelissen JJ, van Putten WL, Verdonck LF, Theobald M, Jacky E, Daenen SM, et al. Results of a HOVON/ SAKK donor versus no-donor analysis of myeloablative HLA-identical sibling stem cell transplantation in first remission acute myeloid leukemia in young and midd- 
le-aged adults: benefits for whom? Blood 2007; 109 (9): 3658-66.

2. Klepin H, Ritchie E, Major-Elechi B, Le-Rademacher J, Seisler D , Storrick L, et al. Geriatric assessment among older adults receiving intensive therapy for acute myeloid leukemia: report of CALGB 361006 (Alliance). J Geriatr Oncol 2020; 11 (1): 107-13.

3. Levin-Epstein R, Oliai C, Schiller G. Allogeneic hematopoietic stem cell transplantation for older patients with acute myeloid leukemia. Curr Treat Options Oncol 2018; 19 (12): 63.

4. Ossenkoppele G, Löwenberg B. How I treat the older patient with acute myeloid leukemia. Blood 2015; 125 (5): 767-74.

5. http://www.bibliotecaminsal.cl/wp/wp-content/ uploads/2016/04/Leucemia-15-a\%C3\%B1os-y-m\%C3\%A1s.pdf - página 22.

6. Sarmiento MM, Bertín CP, Jara V, Ocqueteau TM, Ramírez VP. Ajuste de intensidad para el trasplante hematopoyético alogénico en leucemia aguda. Rev Med Chile 2016; 144 (9): 1112-8.

7. Sarmiento M, Ramirez P, Jara V, Bertin P, Galleguillos M, Rodriguez I, et al. Haploidentical transplantation outcomes are comparable with those obtained with identical human leukocyte antigen allogeneic transplantation in Chilean patients with benign and malignant hemopathies. Hematol Transfus Cell Ther 2020; 42 (1): 40-5.

8. Thomas CM, Ippoliti C, Roboz GJ, Feldman E, Savva D, James S, et al. Clofarabine as a bridge to hematopoietic stem cell transplant. Leuk Lymphoma 2017; 58 (1): 2302. doi: 10.1080/10428194.2016.1185784.

9. Koreth J, Schlenk R, Kopecky KJ, Honda S, Sierra J, Djulbegovic BJ, et al. Allogeneic stem cell transplantation for acute myeloid leukemia in first complete remission: systematic review and meta-analysis of prospective clinical trials. JAMA 2009; 301 (22): 2349-61.

10. Ma S, Shi W, Li Z, Ma S, Shi W, Ziying L, et al. Redu- ced-intensity versus Myeloablative Conditioning Regimens for Younger Adults with Acute Myeloid Leukemia and Myelodysplastic Syndrome: A systematic review and meta-analysis. J Cancer 2020; 11 (17): 5223-35. Published 2020 Jul 6. doi:10.7150/jca.46081.

11. Cornelissen JJ, van Putten WL, Verdonck LF, Theobald M, Jacky E, Daenen SM, et al. Results of a HOVON/ SAKK donor versus no-donor analysis of myeloablative HLA-identical sibling stem cell transplantation in first remission acute myeloid leukemia in young and middle-aged adults: benefits for whom? Blood 2007; 109 (9): 3658-66.

12. Santoro N, Labopin M, Ciceri F, Van Lint MT, Nasso $\mathrm{D}$, Blaise $\mathrm{D}$, et al. Impact of conditioning intensity on outcomes of haploidentical stem cell transplantation for patients with acute myeloid leukemia 45 years of age and over. Cancer 2019; 125 (9): 1499-1506. doi:10.1002/ cncr.31941.

13. Ringdén O, Boumendil A, Labopin M, Canaani J, Beelen D, Ehninger G, et al. Outcome of allogeneic hematopoietic stem cell transplantation in patients age \&gt;69 years with acute myelogenous leukemia: on behalf of the acute leukemia working party of the european society for blood and marrow transplantation. Biol Blood Marrow Transplant 2019; 25 (10): 1975-83.

14. Ustun C, Le-Rademacher J, Wang HL, Othus M, Sun $\mathrm{Z}$, Major B, et al. Allogeneic hematopoietic cell transplantation compared to chemotherapy consolidation in older acute myeloid leukemia (AML) patients 60-75 years in first complete remission (CR1): an alliance (A151509), SWOG, ECOG-ACRIN, and CIBMTR study. Leukemia 2019; 33 (11): 2599-609.

15. Ciurea SO, Kongtim P, Varma A, Rondon G, Chen J, Srour S, et al. Is there an optimal conditioning for older patients with AML receiving allogeneic hematopoietic cell transplantation? Blood. 2019 Dec 11:2019003662. Epub ahead of print. DOI:10.1182/ blood.2019003662. 